\title{
Corrigendum to "Simulation-Based Early Prediction of Rocket, Artillery, and Mortar Trajectories and Real-Time Optimization for Counter-RAM Systems"
}

\author{
Arash Ramezani $(\mathbb{D})$ and Hendrik Rothe \\ Helmut-Schmidt-University/University of the Federal Armed Forces Hamburg, Institute of Automation Technology, \\ Chair of Measurement and Information Technology, Holstenhofweg 85, 22043 Hamburg, Germany
}

Correspondence should be addressed to Arash Ramezani; ramezani@hsu-hh.de

Received 4 June 2019; Accepted 4 June 2019; Published 24 June 2019

Copyright (C) 2019 Arash Ramezani and Hendrik Rothe. This is an open access article distributed under the Creative Commons Attribution License, which permits unrestricted use, distribution, and reproduction in any medium, provided the original work is properly cited.

In the article titled "Simulation-Based Early Prediction of Rocket, Artillery, and Mortar Trajectories and Real-Time Optimization for Counter-RAM Systems" [1], there was an error in Section 2.1, where the fourth and fifth paragraphs, "The air drag coefficient $C_{\_} d$ for instance depends on the critical velocity ratio, pictured in Figure 1 . Since the drag coefficient does not vary in a simple manner with Mach number, this makes the analytic solutions inaccurate and difficult to accomplish." and "One can see from this figure that there is no simple analytic solution to this variation. With computer power nowadays, we usually solve or approximate the exact solutions numerically, doing the quadratures by breaking the area under the curve into quadrilaterals and summing the areas. In general, there are three forms of the drag coefficient:", respectively, should be updated to the following:

Fourth paragraph: "The air drag coefficient $C_{-} d$ for instance depends on the critical velocity ratio, pictured in Figure 1. Due to Carlucci and Jacobson [19], these analytic solutions are hard to achieve and prone to error, because the drag coefficient does not differ in a straightforward way with Mach number."

Fifth paragraph: "It is obvious that there is no simple analytical solution for these variants. With modern computer systems, the exact solutions are solved numerically, doing the quadratures by breaking the area under the curve into quadrilaterals and summing the areas. According to Carlucci and Jacobson [19], there are three forms of the drag coefficient:"

\section{References}

[1] A. Ramezani and H. Rothe, "Simulation-based early prediction of rocket, artillery, and mortar trajectories and real-time optimization for counter-ram systems," Mathematical Problems in Engineering, vol. 2017, Article ID 8157319, 8 pages, 2017. 


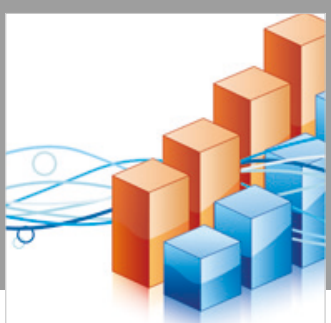

Advances in

Operations Research

\section{-n-m}
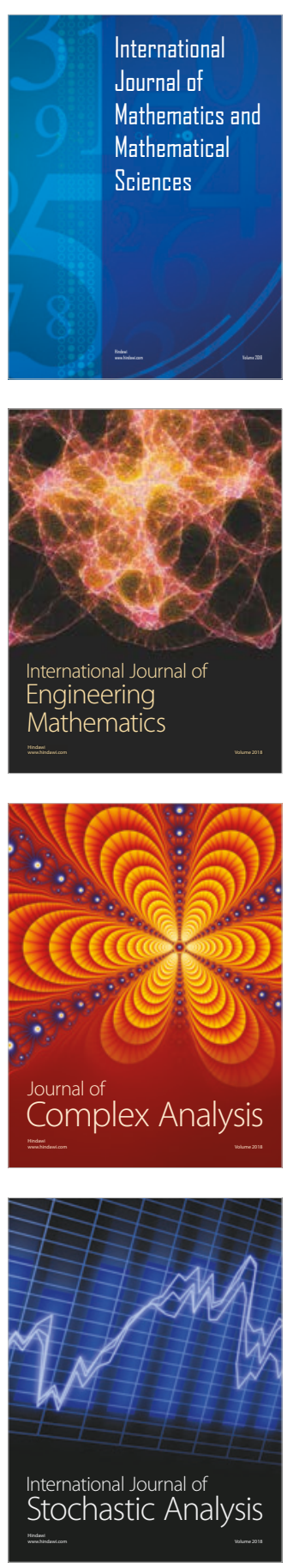
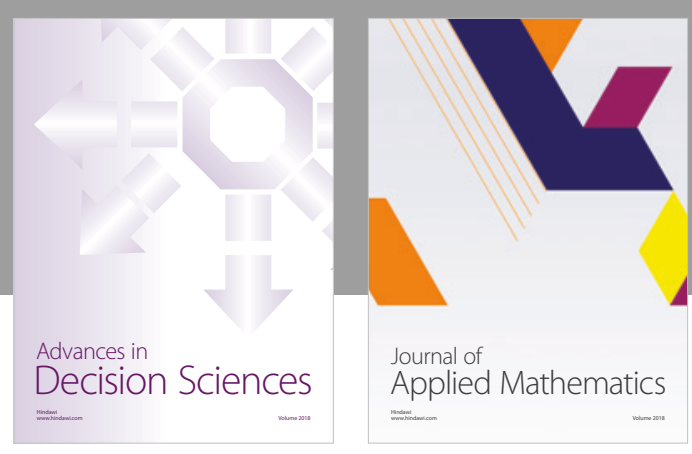

Journal of

Applied Mathematics
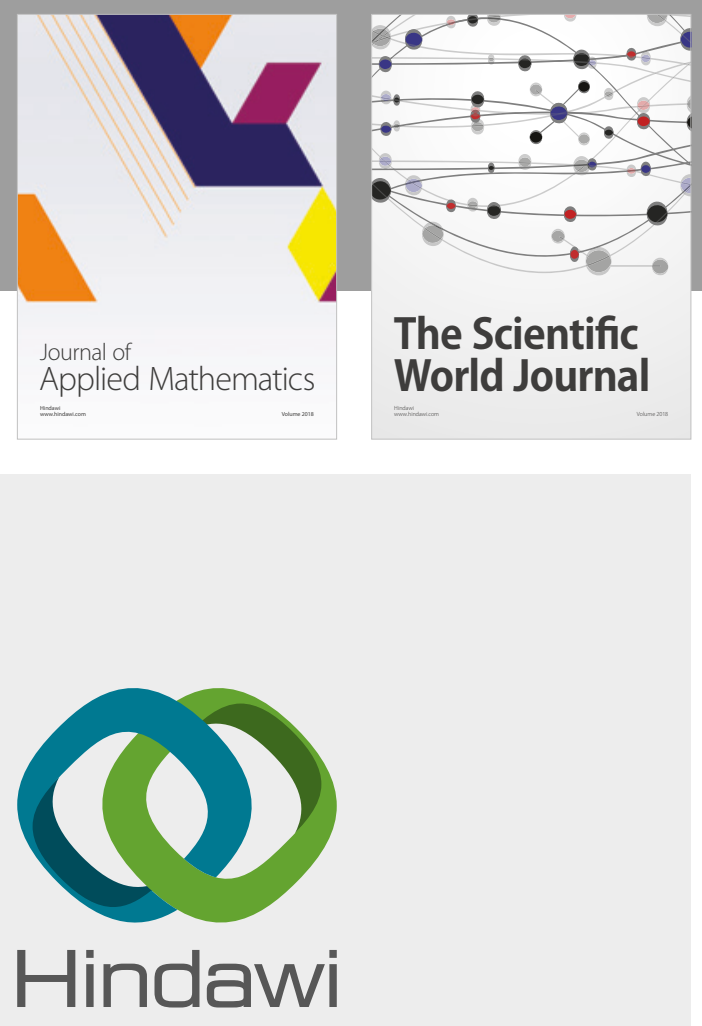

Submit your manuscripts at

www.hindawi.com

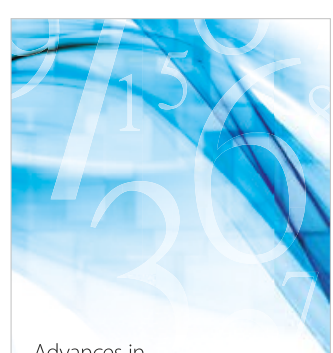

Advances in
Numerical Analysis
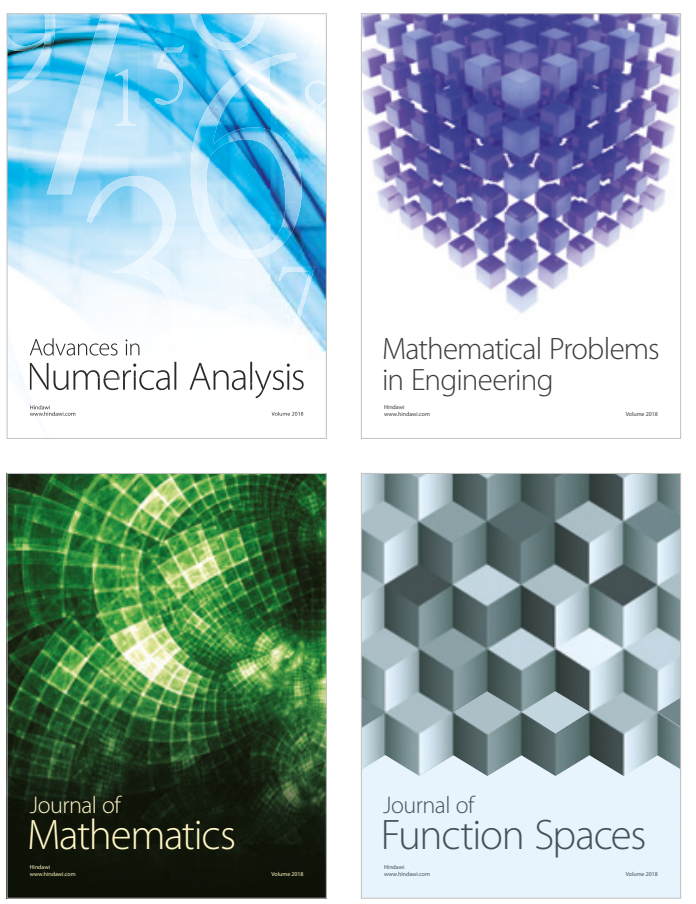

Mathematical Problems in Engineering

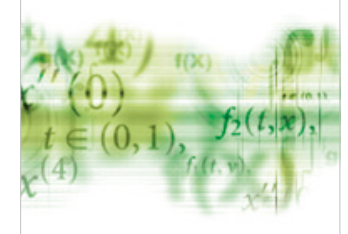

International Journal of

Differential Equations

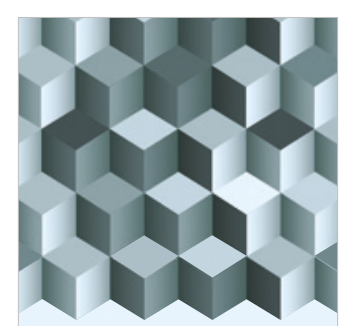

Journal of

Function Spaces
The Scientific

World Journal

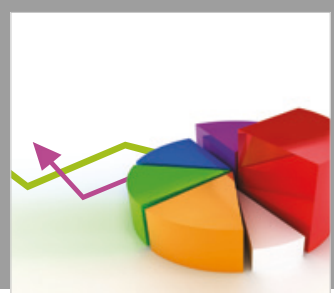

Journal of

Probability and Statistics
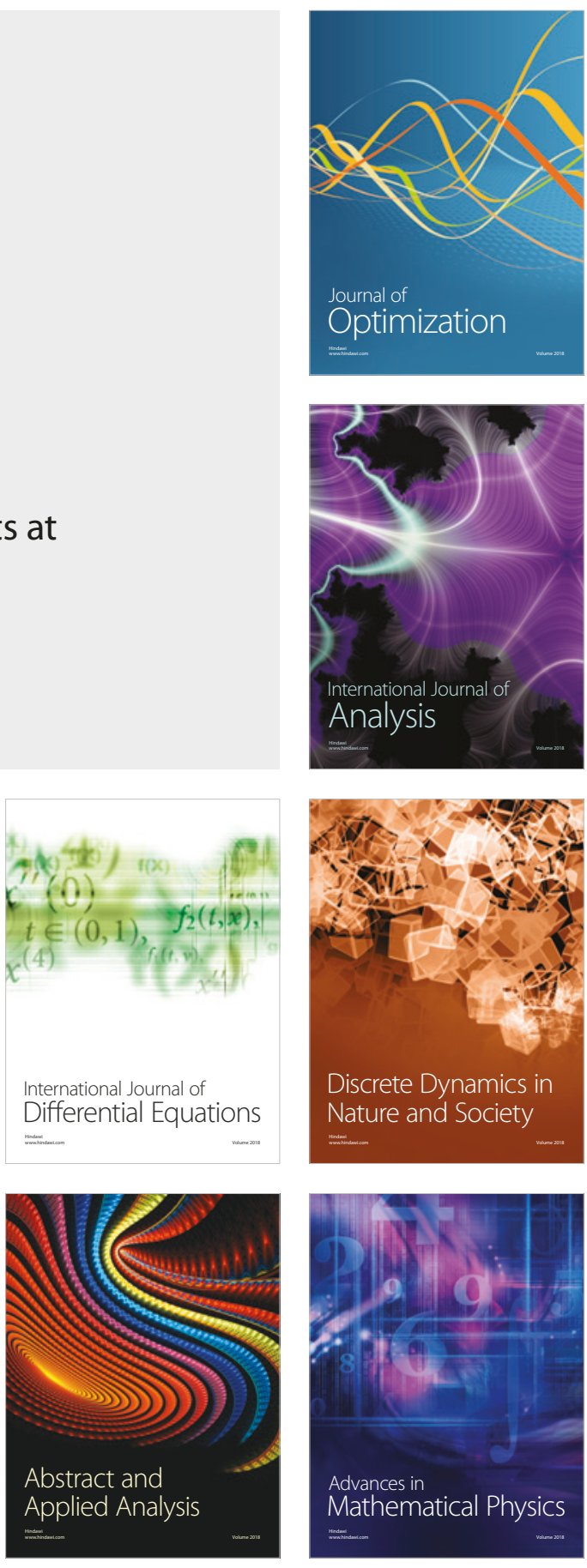